\title{
Modeling Interactions and Shoaling of Solitary Waves Using a Hybrid Finite Volume and Finite Difference Solver
}

\author{
Burak Turan ${ }^{1}$ and Keh-Han Wang ${ }^{2 *}$ \\ ${ }^{1}$ Emek 4, Cad No: 158/4, Cankaya, Ankara, Turkey \\ ${ }^{2}$ Department of Civil and Environmental Engineering, University of Houston, Houston, TX 77204-4003, USA
}

\begin{abstract}
This paper presents a mixed finite volume and finite difference solver with results showing the solitary wave interactions and shoaling process by solving a set of conservative forms of Boussinesq equations. A second order accurate finite volume scheme is applied to the conservative terms of the governing equations while up to the second order finite difference formulations are used to discretize the dispersive source terms with higher order derivatives. The limiters and surface gradient method are implemented in the model to remove the unwanted spurious oscillations and preserve the still water condition without introducing errors at the interfaces. The performance of the present numerical solver is tested with results of head on collisions and shoaling of solitary waves compared against those from finite element models that were developed based on fully nonlinear weakly dispersive and weakly nonlinear weakly dispersive forms of the Boussinesq equations as well as analytical solutions and experimental observations.
\end{abstract}

Keywords: Solitary wave; Shoaling, Finite volume; Finite difference; Boussinnesq Equations

\section{Introduction}

Modeling the propagation and shoaling transformation of solitary waves in shallow water regions is practically important to the study of impacts of nonlinear waves on coastal environments. Especially, the prediction of wave run up caused by a solitary wave like tsunami plays critical role in the planning of coastal protection. Therefore, studies of solitary waves experimentally and numerically have been performed to characterize the tsunami wave movement towards the coastal regions. In the 1870's, Boussinesq and Rayleigh described solitary waves mathematically with the so-called Bousinessq equations (BE) [1]. Madsen et al. [2] developed a new form of BE to improve the linear dispersion characteristics of the BE in deeper water. Later, Madsen and Sorensen [3] generalized this new form of the BE for mild sloped bottom in order to model shoaling of waves from deep to shallow water. Introducing the layer-mean velocity potential, $\mathrm{Wu}$ [4] developed a set of generalized $\mathrm{BE}$ for modeling nonlinear shallow water waves. Nwogu [5] also derived a different form of BE to approximate wave propagation at much deeper environment. The finite difference (FD) schemes have been extensively used to discretize the nonlinear wave equations $[2,6-8]$. While modeling complex geometries, finite element (FE) method allows the computational meshes matched closely to the flow domain and obtain more accurate results. Zhong and Wang [9] stated that spurious oscillations adversely affect the numerical results when convection dominates in wave propagation. It was known that the Godunov [10] type finite volume (FV) schemes have the superiority in capturing flow discontinuities or oscillations in regular or irregular domains [11-13]. Therefore, application of FV numerical method to discretize $\mathrm{BE}$ has attracted researchers' attention. The combined FVFD schemes were developed [14-17] to discretize the BE, where the FV method was applied to discretize the conservative parts and the FD scheme to discretize the dispersive terms and source terms. Stansby [14] modeled solitary wave run-up cases using FV-FD hybrid scheme. Erduran et al. [15] tested the hybrid scheme by simulating propagation of sinusoidal waves in deep and shallow water and their propagation over a submerged bar. Modeling propagation of regular waves and random waves along a sloping bottom was performed by Shiachet and Mingham [16]. Investigation of wave propagation, shoaling and runup using a finite volume solver of the Boussinesq equations was performed recently by Kazolea et al. [18]. Zhong and Wang [9] pointed out that the researchers had concentrated more on modeling monochromatic deep water waves, but nonlinear shallow-water waves such as solitary waves and their diffraction and refraction in domains with variable bottom topography had not been studied extensively. Therefore, this paper focuses on modeling cases of solitary wave transformation by discretizing the improved form of BE with a developed finite volumefinite difference solver. Nonlinear wave-wave interaction can be tested by simulating the collision of two solitary waves. During the collision of two solitary waves, waves behave like interacting elementary particles such as electrons or protons [19]. Byatt-Smith [20] derived a formulation showing that the peak of the interaction after head-on collision of two equal-amplitude waves is greater than twice of the initial wave amplitude. Yih and $\mathrm{Wu}$ [21] derived a corrected version of BE for long water waves and proposed a general solution for the interaction of solitary waves. Hammack et al., [22] investigated both the following and counter propagating (head-on) collisions by numerically solving Euler's equations. As solitary waves approximately resemble steep waves on beaches, the shoaling and runup of solitary waves on a sloping bottom is a main concern in coastal regions [23]. Shoaling process can be calculated up to the breaking point based on weakly nonlinear wave theories [23]. The bed slope gradient of the cells becomes more important while discretizing flow equations on a computational slope with variable bottom topography. Centered discretization of slope term fails when the waves with sharp wave front propagate on nonflat bed. Zhou et al. [24] suggested the surface gradient method (SGM) to overcome this difficulty. In the SGM, the reconstruction of water

${ }^{*}$ Corresponding author: Keh-Han Wang, Deptartment of Civil and Environmental Engineering, University of Houston, Houston, TX 77204-4003, USA, Tel: 1-713743-4277; Fax: 1-713-743-4260; E-mail: khwang@uh.edu

Received May 24, 2014; Accepted July 09, 2014; Published July 14, 2014

Citation: Wang KH, Turan B (2014) Modeling Interactions and Shoaling of Solitary Waves Using a Hybrid Finite Volume and Finite Difference Solver. J Appl Computat Math 3: 173 doi:10.4172/2168-9679.1000173

Copyright: $\odot 2014$ Wang KH, et al. This is an open-access article distributed under the terms of the Creative Commons Attribution License, which permits unrestricted use, distribution, and reproduction in any medium, provided the original author and source are credited. 
surface level rather than water depth is implemented to get rid of the spurious waves and preserve the still water condition without creating errors at the interfaces. A combined finite volume and finite difference model by solving the Madsen et al., [3] BE with a second order accurate FV scheme applied to the conservative terms and FD scheme on the dispersive source terms is developed in this study to model propagation of solitary waves, head-on collisions of solitary waves, and solitary wave shoaling over a submerged shoal. The SGM proposed by Zhou et al. [24] is included in the present study to overcome numerical errors during propagation of waves along variable topography. Zhong and Wang $[9,25]$ developed finite element solvers to discretize fully nonlinear weakly dispersive (FNWD) and weakly nonlinear weakly dispersive (WNWD) forms of the BE. The results obtained from the present FVFD model are compared with those from the FE solvers developed by Zhong and Wang $[9,25]$ and other published solutions.

\section{Governing Equations}

The one-dimensional (1-D) Boussinesq equations proposed by Madsen et al., [3] for waves propagating over regions of shallow water depth (Figure 1) are expressed as

$$
\begin{aligned}
& \frac{\partial \zeta}{\partial t}+\frac{\partial(u h)}{\partial x}=0 \\
& \frac{\partial(u h)}{\partial t}+\frac{\partial}{\partial x}\left(u^{2} h\right)+g h \frac{\partial \zeta}{\partial x}+D+g h S_{f x}=0
\end{aligned}
$$

where

$\zeta=$ free-surface elevation measured from the still water surface level,

$\mathrm{h}=$ water depth measured from the bottom of the channel to the water surface level,

$\mathrm{u}=$ velocity in $\mathrm{x}$ direction,

$\mathrm{S}_{\mathrm{fx}}=$ friction slope in $\mathrm{x}$ direction, and

$\mathrm{D}=$ dispersive term, which is given as

$$
D=-\left(B+\frac{1}{3}\right) H^{2}\left(\frac{\partial^{3} q}{\partial x^{2} \partial t}\right)-B g H^{3}\left(\frac{\partial^{3} \zeta}{\partial x^{3}}\right)-H \frac{\partial H}{\partial x}\left(\frac{1}{3} \frac{\partial^{2} q}{\partial x \partial t}+2 B g H \frac{\partial^{2} \zeta}{\partial x^{2}}\right)
$$

where $\mathrm{h}=\mathrm{H}+\zeta, \mathrm{q}=\mathrm{uh}$, and $\mathrm{B}=$ dispersion coefficient. As suggested by Madsen and Sorensen [3], $B=1 / 15$. Replacing the derivatives of freesurface elevation $\zeta$ with the derivatives of water depth $\mathrm{h}$, we have from Eqs. (1) and (2) the revised Boussinesq equations (BE) as

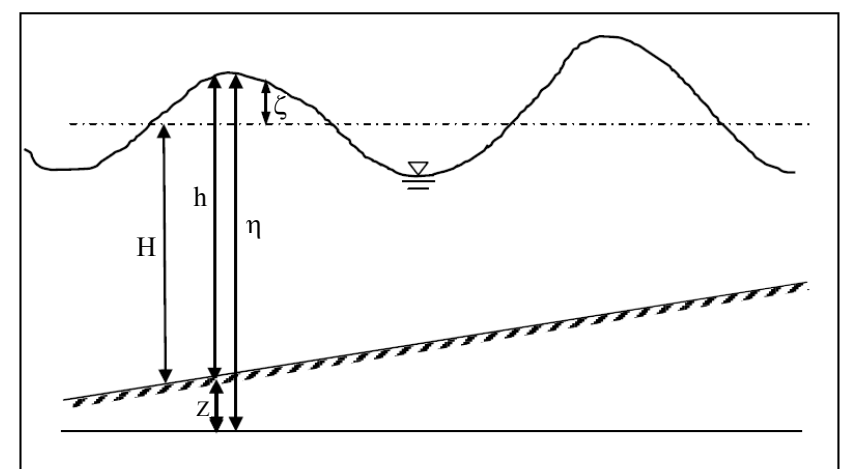

Figure 1: Schematic diagram of wave propagation in a domain of uneven bottom.

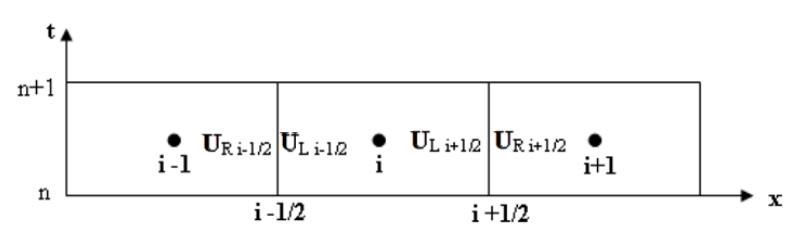

Figure 2: A section of meshes used in FV-FD scheme and conserved variables at the left and right cell edges.

$$
\begin{aligned}
& \frac{\partial h}{\partial t}+\frac{\partial u h}{\partial x}=0 \\
& \frac{\partial u h}{\partial t}+\frac{\partial}{\partial x}\left(u^{2} h+\frac{1}{2} g h^{2}\right)+D=g h\left(S_{o x}-S_{f x}\right)
\end{aligned}
$$

\section{Hybrid Finite Volume and Finite Difference Formulations}

The time and spatial discretization using a predictor-corrector type scheme for the 1-D BE on a small section of the computational domain defined in Figure 2 is given as

$$
\begin{aligned}
& \mathrm{U}_{\mathrm{i}}^{\mathrm{p}}=\mathrm{U}_{\mathrm{i}}^{\mathrm{n}}-\frac{\Delta \mathrm{t}}{2}\left[\frac{\mathrm{F}_{\mathrm{i}+1 / 2}^{\mathrm{n}}-\mathrm{F}_{\mathrm{i}-1 / 2}^{\mathrm{n}}}{\Delta \mathrm{x}}\right]+\frac{\Delta \mathrm{t}}{2} \mathrm{~S}_{\mathrm{i}}^{\mathrm{n}} \\
& \mathrm{U}_{\mathrm{i}}^{\mathrm{n}+1}=\mathrm{U}_{\mathrm{i}}^{\mathrm{n}}-\Delta \mathrm{t}\left[\frac{\mathrm{F}_{\mathrm{i}+1 / 2}^{\mathrm{p}}-\mathrm{F}_{\mathrm{i}-1 / 2}^{\mathrm{p}}}{\Delta \mathrm{x}}\right]+\Delta \mathrm{tS}_{\mathrm{i}}^{\mathrm{p}}
\end{aligned}
$$

Where

$$
\mathrm{U}=\left[\begin{array}{l}
\mathrm{u} \\
\mathrm{uh}
\end{array}\right], \mathrm{F}=\left[\begin{array}{c}
\mathrm{uh} \\
\mathrm{u}^{2} \mathrm{~h}+\frac{1}{2} \mathrm{gh}^{2}
\end{array}\right] \text {, and } \mathrm{S}_{\mathrm{i}}^{\mathrm{n}}=\left[\begin{array}{c}
0 \\
\mathrm{gh}\left(\mathrm{s}_{\mathrm{ox}}-\mathrm{s}_{\mathrm{fx}}\right)-\mathrm{D}
\end{array}\right]_{\mathrm{i}}^{\mathrm{n}},
$$

$\mathbf{U}=$ vector of 1-D conserved variables,

$\mathbf{F}=$ vector of 1-D flux function,

$\mathbf{S}=$ vector of 1-D source terms.

$\mathrm{D}=$ dispersive term,

Superscript $n, p$, and $n+1=$ variables at $n$ time level, predictor level and $\mathrm{n}+1$ time level,

$\mathrm{S}_{\mathrm{ox}}=$ the bottom slope of the channel in $\mathrm{x}$ direction, $-\partial \mathrm{z} / \partial \mathrm{x}$,

$\mathrm{S}_{\mathrm{fx}}=$ friction slope in $\mathrm{x}$ direction,

$\mathrm{z}=$ bottom elevation,

$\Delta \mathrm{x}, \Delta \mathrm{t}=$ mesh size and time step,

and $n=$ Manning's roughness coefficient.

To have more accurate solutions, the Roe [26] second-order scheme is used. Different from the first-order scheme using directly the values at cell centers, the conserved variables are first reconstructed at the left and right sides of cell edges using the limiters and surface gradient method. As depicted in Figure 2, the position immediately inside of each cell edge, which is being updated, is named as the left side (L) and outside as the right side (R). The flux vectors at the cell interfaces of cell $\mathrm{i}, \mathbf{F}_{i \pm 1 / 2}$, can be discretized as

$$
\mathrm{F}_{\mathrm{i} \pm 1 / 2}=\frac{1}{2}\left[\mathrm{~F}\left(\mathrm{U}_{\mathrm{L}}\right)_{\mathrm{i} \pm 1 / 2}+\mathrm{F}\left(\mathrm{U}_{\mathrm{R}}\right)_{\mathrm{i} \pm 1 / 2}-(|\overline{\mathbf{J}}| \Delta \mathrm{U})_{\mathrm{i} \pm 1 / 2}\right]
$$


Where $\overline{\mathbf{J}}=$ Jacobian matrix and

$$
(|\bar{J}| \Delta U)_{\mathrm{i} \pm 1 / 2}=\sum_{\mathrm{m}=1}^{2}\left|\bar{\lambda}^{\mathrm{m}}\right|_{\mathrm{i} \pm 1 / 2} \overline{\mathrm{W}}_{\mathrm{i} \pm 1 / 2}^{\mathrm{m}}
$$

InEq. (9), wehave $\overline{\mathrm{W}}_{\mathrm{i \neq 1/2}}^{\mathrm{m}}=\alpha_{\mathrm{i} 1 / 2}^{\mathrm{m}} \overline{\mathrm{T}}_{ \pm 1 / 2}^{\mathrm{m}}$ and $\alpha_{\mathrm{i} 1 / 2}^{\mathrm{m}}=\left(\mathrm{R}^{-1} \Delta \mathrm{U}\right)_{\mathrm{i} \pm 1 / 2}^{\mathrm{m}}(\mathrm{m}=1,2), \mathrm{R}^{-1}=$ left eigenvector matrix, $\bar{r}=$ eigenvectors, and $\bar{\lambda}=$ averaged eigenvalues representing the speeds of characteristics. The average states of water depth, velocity, and eigenvectors are computed with the formulas shown below [26]

$$
\begin{aligned}
& \overline{\mathrm{h}}_{i \pm 1 / 2}=\left(h_{i}+h_{i \pm 1}\right) / 2 \\
& \overline{\mathrm{U}}_{\mathrm{i} \pm 1 / 2}=\left(\sqrt{\mathrm{h}_{\mathrm{i}}} \mathrm{u}_{\mathrm{i}}+\sqrt{\mathrm{h}_{\mathrm{i} \pm 1}} \mathrm{u}_{\mathrm{i} \pm 1}\right) / \sqrt{\mathrm{h}_{\mathrm{i}}+\mathrm{h}_{\mathrm{i} \pm 1}} \\
& \overline{\mathbf{r}}_{\mathrm{i} \pm 1 / 2}^{1}=\left[\begin{array}{l}
1 \\
\bar{\lambda}_{\mathrm{i} \pm 1 / 2}^{1}
\end{array}\right] \\
& \overline{\mathbf{r}}_{\mathrm{i} \pm 1 / 2}^{2}=\left[\begin{array}{l}
1 \\
\bar{\lambda}_{\mathrm{i} \pm 1 / 2}^{2}
\end{array}\right]
\end{aligned}
$$

where $\bar{\lambda}_{\mathrm{i} \pm 1 / 2}^{1}=\overline{\mathrm{u}}_{\mathrm{i}+1 / 2}-\sqrt{\mathrm{g}_{\mathrm{i}+1 / 2}}$ and $\bar{\lambda}_{\mathrm{i} \pm 1 / 2}^{2}=\overline{\mathrm{u}}_{\mathrm{i}+1 / 2}+\sqrt{\mathrm{gh}_{\mathrm{i} 11 / 2}}$. It should be noted that reconstruction of the water surface level rather than the water depth at the interface provides more accurate and stable calculations when the bottom is non-flat. The water surface levels $\eta(\eta=h+z)$ at left and right sides of the interface are calculated by using the following equations $[27,28]$

$$
\begin{aligned}
& \eta_{i \pm 1 / 2}^{L}=\eta_{\mathrm{i}} \pm \frac{1}{2}{\overline{\Delta \eta_{\mathrm{i}}}} \\
& \eta_{i \pm 1 / 2}^{R}=\eta_{\mathrm{i} \pm 1} \pm \frac{1}{2} \overline{\Delta \eta}_{\mathrm{i} \pm 1}
\end{aligned}
$$

where $\overline{\Delta \eta}_{\mathrm{i}}, \overline{\Delta \eta}_{\mathrm{i}-1}$, and $\overline{\Delta \eta}_{\mathrm{i}+1}$ are respectively the limited gradients of cells $\mathrm{i}, \mathrm{i}-1$, and $\mathrm{i}+1$.

$\eta_{i \pm 1 / 2}^{R}$ and $\eta_{i \pm 1 / 2}^{L}$ are the constructed water surface elevation at the right and left sides of cell edges of $i+1 / 2$ and $i-1 / 2$. Similarly, the discharge $\mathrm{q}$ is reconstructed at the edges using the same formulations by replacing $\eta$ by q. The Double Minmod (DB) limiter [27,29] is used and integrated in the present numerical solver to limit the oscillations. The values of $\overline{\Delta \eta}_{\mathrm{i}}$ is calculated as follows:

$$
\begin{aligned}
& \text { if } \Delta \eta_{\mathrm{i}-1 / 2} \Delta \eta_{\mathrm{i}+1 / 2}>0 \\
& \overline{\Delta \eta}_{\mathrm{i}}=\operatorname{Sign}\left(\Delta \eta_{\mathrm{i}+1 / 2}\right) \min \left(\frac{1}{2}\left|\Delta \eta_{\mathrm{i}-1 / 2}+\Delta \eta_{\mathrm{i}+1 / 2}\right|, 2\left|\Delta \eta_{\mathrm{i}-1 / 2}\right|, 2\left|\Delta \eta_{\mathrm{i}+1 / 2}\right|\right)
\end{aligned}
$$

Otherwise $\overline{\Delta \eta_{i}}=0$,

Where

$$
\begin{aligned}
& \Delta \eta_{i-1 / 2}=\eta_{i}-\eta_{i-1} \\
& \Delta \eta_{i+1 / 2}=\eta_{i+1}-\eta_{i}
\end{aligned}
$$

As described by van Leer [29] the Double Minmod algorithm in Eq. (13) limits the algebraic average of $\Delta \eta_{i-1 / 2}$ and $\Delta \eta_{i+1 / 2}$ to twice of the smaller one. In comparison with the Superbee limiter $[29,30]$, Bradford and Sanders [28] indicated that the DM limiter yields a better performance than the Superbee. Once $\eta_{i \pm 1 / 2}^{L}$ and $\eta_{i \pm 1 / 2}^{R}$ are determined, water depths at the edges (for example, at $\mathrm{i}+1 / 2$ ) are computed using

$$
\begin{aligned}
& \mathrm{h}_{i+1 / 2}^{L}=\eta_{i+1 / 2}^{L}-z_{i+1 / 2} \\
& \mathbf{h}_{i+1 / 2}^{R}=\eta_{i+1 / 2}^{R}-z_{i+1 / 2}
\end{aligned}
$$

The bottom slope term in Eq. (5) is computed using

$$
\left(\mathrm{ghS}_{\mathrm{ox}}\right)_{\mathrm{i}}=\mathrm{gh}_{\mathrm{i}}\left(-\frac{\partial \mathrm{z}}{\partial \mathrm{x}}\right)_{\mathrm{i}}=\mathrm{gh}_{\mathrm{i}}\left(\frac{\partial \mathrm{H}}{\partial \mathrm{x}}\right)_{\mathrm{i}}=\mathrm{gh}_{\mathrm{i}} \frac{\mathrm{H}_{\mathrm{i}+1 / 2}-\mathrm{H}_{\mathrm{i}-1 / 2}}{\Delta \mathrm{x}}
$$

to preserve the balance between the flux and slope terms at stationary flow conditions. Including the finite difference schemes on the time and spatial discretization of the dispersive terms in Eq. (3) into the finite volume formulations of the conservative and source terms, the predictor formulation of the momentum equation (Eq. (6)) with unknown variable of $\mathrm{q}(\mathrm{q}=\mathrm{uh})$ is given as

$$
\mathrm{V}_{1} \mathrm{q}_{\mathrm{i}+1}^{\mathrm{p}}+\mathrm{W}_{1} \mathrm{q}_{\mathrm{i}}^{\mathrm{p}}+\mathrm{X}_{1} \mathrm{q}_{\mathrm{i}-1}^{\mathrm{p}}=\text { RHS }
$$

where

$$
\begin{aligned}
& \mathrm{RHS}=\mathrm{V}_{1} \mathrm{q}_{\mathrm{i}+1}^{\mathrm{n}}+\mathrm{W}_{1} \mathrm{q}_{\mathrm{i}}^{\mathrm{n}}+\mathrm{X}_{1} \mathrm{q}_{\mathrm{i}-1}^{\mathrm{n}}-\frac{\Delta \mathrm{t}}{2} \mathrm{R}_{\mathrm{i}}^{\mathrm{n}} \\
& \mathrm{V}_{1}=\mathrm{K}+\mathrm{L} \\
& \mathrm{W}_{1}=1-2 \mathrm{~K} \\
& \mathrm{X}_{1}=\mathrm{K}-\mathrm{L} \\
& \mathrm{K}=-\left(\mathrm{B}+\frac{1}{3}\right) \frac{\mathrm{H}_{\mathrm{i}}^{\mathrm{n}^{2}}}{\Delta \mathrm{x}^{2}} \\
& \mathrm{~L}=-\frac{1}{12} \mathrm{H}_{\mathrm{i}}^{\mathrm{n}} \frac{\mathrm{H}_{\mathrm{i}+1}^{\mathrm{n}}-\mathrm{H}_{\mathrm{i}-1}^{\mathrm{n}}}{\Delta \mathrm{x}^{2}} \\
& \mathrm{R}_{\mathrm{i}}{ }^{\mathrm{n}}=\left[\left(\frac{\mathrm{F}_{\mathrm{i}+1 / 2}^{\mathrm{n}}{ }^{[2]}-\mathrm{F}_{\mathrm{i}-1 / 2}^{\mathrm{n}}{ }^{[2]}}{\Delta \mathrm{x}}\right)-\mathrm{gh}_{\mathrm{i}}^{\mathrm{n}}\left(\frac{\mathrm{H}_{\mathrm{i}+1 / 2}^{\mathrm{n}}-\mathrm{H}_{\mathrm{i}-1 / 2}^{\mathrm{n}}}{\Delta \mathrm{x}}\right)+\mathrm{gh}_{\mathrm{i}}^{\mathrm{n}} \mathrm{S}_{\mathrm{fi}}{ }^{\mathrm{n}}\right. \\
& -\frac{\mathrm{BgH} \mathrm{H}_{\mathrm{i}}^{\mathrm{n}^{3}}}{2 \Delta \mathrm{x}^{3}}\left[\zeta_{\mathrm{i}+2}^{\mathrm{n}}-2 \zeta_{\mathrm{i}+1}^{\mathrm{n}}+2 \zeta_{\mathrm{i}-1}^{\mathrm{n}}-\zeta_{\mathrm{i}-2}^{\mathrm{n}}\right] \\
& -\frac{\mathrm{BgH} \mathrm{H}_{\mathrm{i}}^{\mathrm{n}^{2}}}{\Delta \mathrm{x}^{3}}\left(\mathrm{H}_{\mathrm{i}+1}^{\mathrm{n}}-\mathrm{H}_{\mathrm{i}-1}^{\mathrm{n}}\right)\left[\zeta_{\mathrm{i}+1}^{\mathrm{n}}-2 \zeta_{\mathrm{i}}^{\mathrm{n}}+\zeta_{\mathrm{i}-1}^{\mathrm{n}}\right]
\end{aligned}
$$

In Eq. (18g), $F_{i \pm 1 / 2}^{n}[2]=$ the second row of the $\mathbf{F}$ vector at the edge $i+1 / 2$ and $i-1 / 2$, respectively. Similarly, the numerical expression for the corrector (Eq. (7)) can also be formulated to compute $q_{i}^{n+1}$.

The finite volume algorithm as a portion of the present hybrid FV-FD solver includes the Roe's second order scheme and an up to $O\left(\Delta \mathrm{x}^{2}\right)$ DM limiter. Here, $\Delta \mathrm{x}$ is the grid size. For the dispersive term, the central differencing scheme, which is also accurate to the order of $\Delta \mathrm{x}^{2}$, is used in the solver. Therefore, with a decrease of $\Delta \mathrm{x}$, converged solutions are obtained. As the present solver adopts the predictorcorrector type solution procedure, Courant condition is utilized to control the stability of the numerical scheme. The condition in terms of the time step $\Delta t$ is given as $\Delta t \leq \Delta x /(\operatorname{Max}(|\overline{\mathrm{u}} \pm \sqrt{g \bar{h}}|))$. With the use of predictor-corrector scheme and the direct solution procedure of tri-diagonal matrix algorithm for Eq. (17), the present FV-FD solver is a cost-effective computational model for simulating propagation and transformation of nonlinear shallow-water waves. 


\section{Simulations of 1-D FV-FD Solver}

The FV-FD solver is tested for cases of head-on collision of two solitary waves with equal or different amplitudes. Comparisons of computed time-varying free-surface elevations are made with other published results and analytical solutions. The simulations for a solitary wave propagating over a domain of sloping bottom are also carried out to investigate the corresponding wave fission and shoaling process. The results of free-surface elevation are compared to the experimental measurements and other published numerical solutions. In all of the simulation cases, transmissive boundary conditions are used both at the upstream and downstream boundaries.

\section{Head-on Collisions of Two Solitary Waves}

The head-on collision of two solitary waves represents the process of two solitons colliding with each other. Similar incident wave conditions as given in Zhong and Wang $[9,25]$ are used. In this test case, the interaction of two solitary waves with identical amplitude, i.e. $a_{1}=a_{2}=0.1 \mathrm{~m}$, is investigated, where $a_{1}$ and $a_{2}$ are initial amplitudes of two propagating solitary waves. The length of the channel is $90 \mathrm{~m}(0 \leq$ $\mathrm{x} \leq 90 \mathrm{~m})$ and the grid size $\Delta \mathrm{x}$ is set as $0.1 \mathrm{~m}$. Zhong and Wang $[9,25]$ developed two dimensional Boussinesq models. One solved the Fully Nonlinear Weakly Dispersive (FNWD) form of the BE and the other solved the Weakly Nonlinear Weakly Dispersive (WNWD) BE using the FE method. The present FV-FD model is simulated for head-on collision of two solitary waves and the results are compared with those computed using the models developed by Zhong and Wang $[9,25]$. The solitary waves are nonlinear shallow-water waves, so, nonlinear interaction results in the maximum amplitude during the collision to be greater than the sum of the two initial wave amplitudes. The peak wave amplitude during the collision process can be derived analytically and was given by Baytt-Smith [20] as

$$
a_{c}=a_{1}+a_{2}+\frac{1}{2} a_{1} a_{2}
$$

where $a_{c}=$ peak wave amplitude during collision. The results obtained from the present solver are compared with those of WNWD and FNWD models by Zhong and Wang $[9,25]$ in Figures 3a-3d. The plots include the time variation of the free-surface elevations before, during and after the collision. Initially, the peak of right-going solitary wave is located at $x=25 \mathrm{~m}$ while the left-going solitary wave is situated at $\mathrm{x}=65 \mathrm{~m}$ (Figure 3a). As can be seen from Figure 3b-Figure 3d, the present solver produces similar results as those from the WNWD and FNWD models. At $t=4.5 \mathrm{sec}$ (Figure $3 \mathrm{~b}$ ), the encountering process of the two solitary waves and partially merged waves can be noticed. The amplitudes of the two collided waves continue increasing until they merge completely into a single solitary wave with a peak amplitude at $\mathrm{t}=6.14 \mathrm{sec}$ (Figure 3c). The formulation in Eq. (19) (Baytt-Smith [20]) gives $0.205 \mathrm{~m}$ as the analytical peak amplitude in this test case. The maximum amplitude after collision is predicted to be at $6.14 \mathrm{sec}$ by all models and the value is $0.2024 \mathrm{~m}$ obtained by the present solver, which is close to the analytical solution. There exists a small phase shift after the interaction of two solitary waves. As depicted in Figure 5d, the merged solitary waves are separated to recover back to their original amplitudes and shapes after colliding with each other. Overall, the results in terms of wave elevations and phases from the present solver agree reasonably well with the simulated solutions from the WNWD and FNWD models and the performance of the solver for capturing the process of head-on collision of two solitary waves is satisfactory. Head-on collision of two solitary waves with different amplitudes is also investigated to further the verification of the solver's capability of simulating interaction of
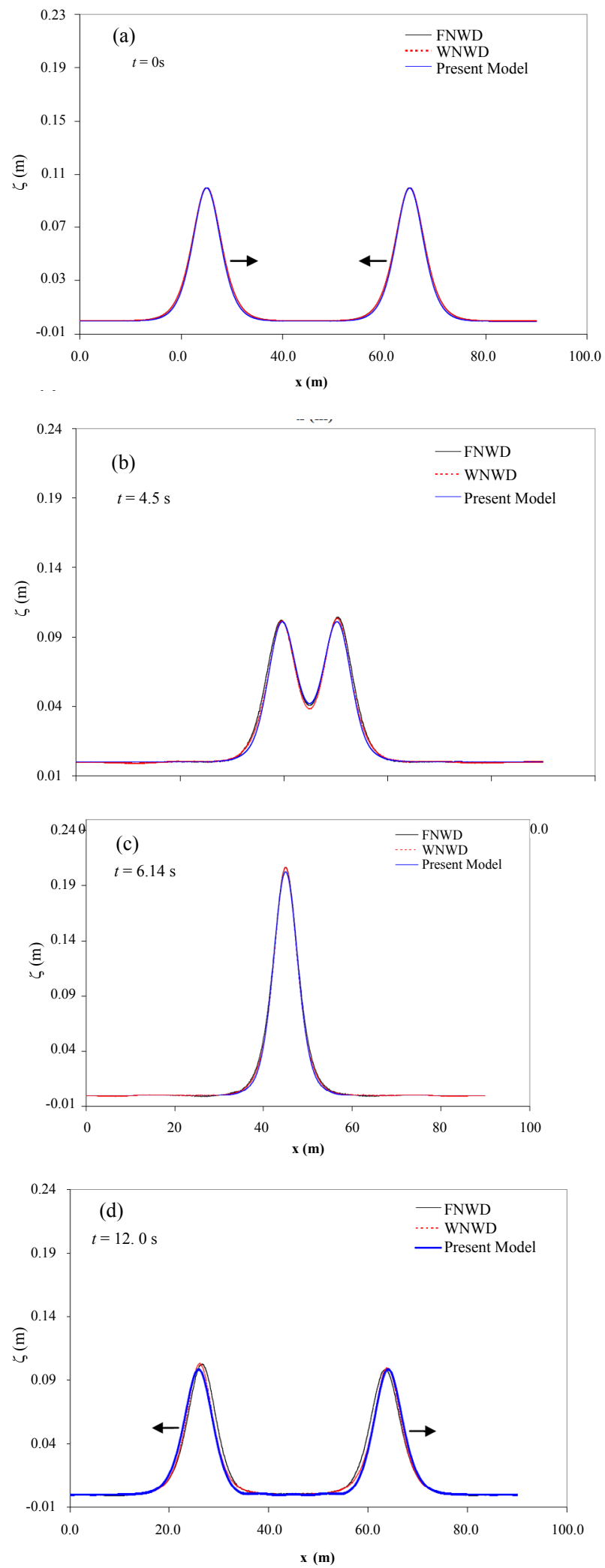

Figure 3: Comparisons of free-surface profiles along $x$ axis for head-on collision of twosolitary waves with equal amplitude of $0.1 \mathrm{~m}$ at $\mathrm{t}=(\mathrm{a}) 0 \mathrm{sec}$, (b) $4.5 \mathrm{sec}$, (c) $6.14 \mathrm{sec}$, (d) $12 \mathrm{sec}$. 


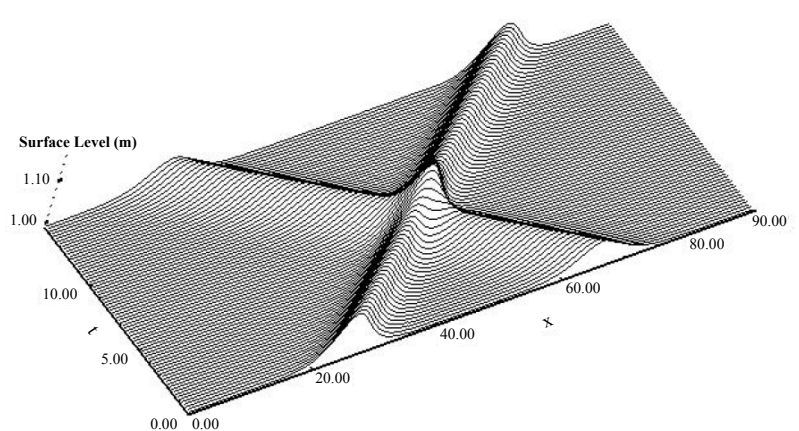

Figure 4: 3D perspective view plot of wave elevation for head-on collision of two solitarywaves with different amplitudes of $0.1 \mathrm{~m}$ and $0.06 \mathrm{~m}$.

nonlinear waves. The domain is the same as the one described above. The amplitudes of the solitary waves located at the left and right sides of the channel initially are $0.1 \mathrm{~m}$ and $0.06 \mathrm{~m}$, respectively. The $3 \mathrm{D}$ perspective view plot showing the evolution of free-surface levels in the entire spatial domain at different times is presented in Figure 4 to provide the visual understanding of the head-on interaction of two solitary waves. According to the analytical formulation given in Eq. (19) the maximum amplitude during the collision of these two waves is calculated as $0.163 \mathrm{~m}$. The peak amplitude and the occurrence time are predicted as $0.162 \mathrm{~m}$ and $6.2 \mathrm{sec}$, respectively by the present model. The predicted peak value is very close to the analytical solution. From Figure 4, we notice that after the interaction, the solitary wave with small amplitude continues to propagate towards the negative $\mathrm{x}$ direction while the larger amplitude wave moves towards the positive $\mathrm{x}$ direction with both waves preserving their original shapes and amplitudes after the collision.

\section{Shoaling of a Solitary Wave over a Sloping Bottom}

At the process of a solitary wave propagating from a region of deeper water depth to that of shallower water depth, the main wave splits into a sequence of waves with decreasing amplitude $[9,25,31,32]$. This is commonly called the fission phenomena of a solitary wave. In this study, two cases of solitary wave shoaling over a sloping bottom are simulated with the developed FV-FD Boussinesq model. The computational domain and changes of water depth selected as the first test case for solitary wave shoaling study are similar to the center line condition of that used by Zhong and Wang $[9,25]$. The dimensionless domain boundaries are from -15 to 60 in $\mathrm{x}$ direction and the dimensionless undisturbed water depth varies from 1 to 0.271 in the shallower water depth region. The water depth variation in dimensionless form (referenced to the deeper water depth) within the domain (also in dimensionless form) utilized is given

$$
\text { as } h(x)= \begin{cases}1 & -15 \leq x \leq 8.335 \\ 1+\frac{1}{11.43}(8.335-x) & 8.335 \leq x \leq 16.667 \\ 0.271 & 16.667 \leq x \leq 60\end{cases}
$$

The dimensionless wave elevations $\zeta / h_{0}$ are plotted along the $\mathrm{x}$ direction at different times in Figure 5 to show the shoaling process as a solitary wave propagates from a region of deeper water depth to a shallower water-depth region. Initially, a solitary wave with dimensionless amplitude of 0.08 is situated at $x=0$ (Figure 5a). As seen in Figure $5 \mathrm{~b}$ when the wave enters the sloping bottom section, a wave with small amplitude is reflected back by the submerged shoal
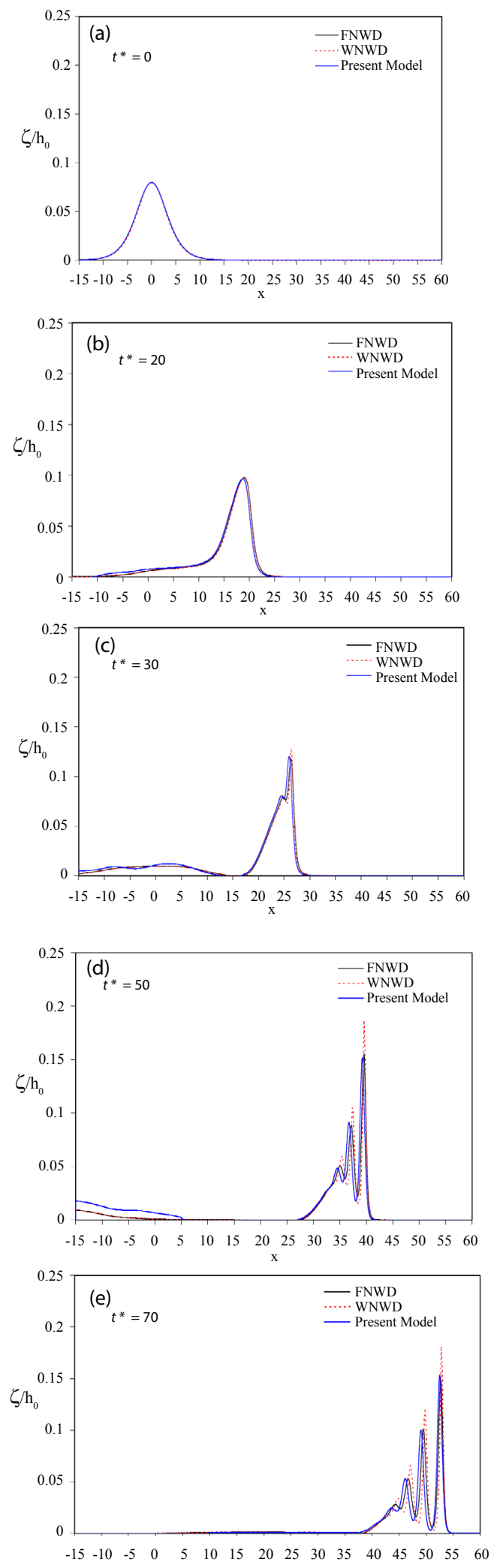

Figure 5:Comparisons of the free-surface profiles obtained from the present model with theWNWD and FNWD models [25] at (a) $t^{*}=0$, (b) $t^{*}=20$, (c) $t^{*}=30$, (d) $t^{*}=50$, and $(e) t^{*}=70$ 


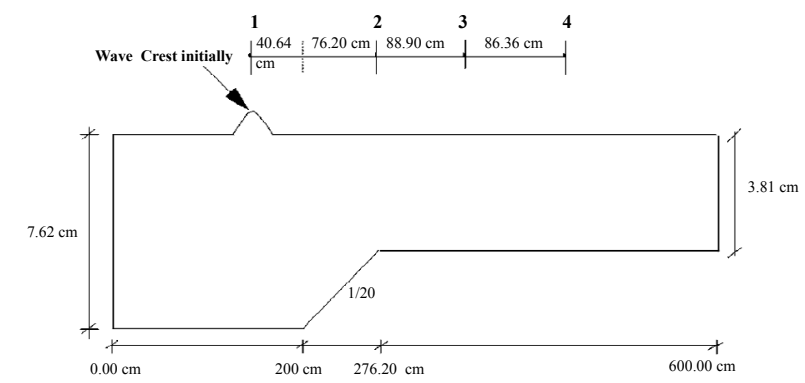

Figure 6: Domain setup and gauging stations [33] considered for modelsimulations and measurement comparisons for a solitary wave propagating over asloping bottom.

to propagate towards the upstream boundary (Figures $5 \mathrm{c}-5 \mathrm{~d}$ ). After the solitary wave enters the submerged shoal region (e.g. at $t^{*}=20$ ), the wave form begins to tilt forward. Here $t^{*}=\sqrt{g h_{0}} t / h_{0}$ and $\mathrm{h}_{0}$ is the water depth in the upstream domain. The fission characteristics are well observed by the transformation of the free surface elevations shown at $t^{*}=30,50$ and 70 . As soon as the main wave propagates past the sloping bottom into the shallower water depth region $\left(t^{*}=30\right)$, the first secondary wave starts to separate from the leading wave. With the main wave traveling further downstream, additional secondary waves with smaller amplitudes are emerged behind the first secondary wave (Figures $5 \mathrm{~d}$ and $5 \mathrm{e}$ ). At $\mathrm{t}^{*}=70$, the interesting wave fission phenomenon reflected by the appearance of three secondary waves with decreasing wave amplitude following the main wave can be noticed. The amplitude of primary wave increases as it propagates in the shallower water depth region towards the downstream boundary. The present model results as shown in Figure 5 match reasonably well with those obtained by Zhong and Wang [25]. Although, a small phase lag is observed at $\mathrm{t}^{*}=50$ and 70 when compared to the WNWD and the FNWD models. In general, the present model results especially the wave peaks fit better with FNWD solutions than the WNWD ones. This suggests the nonlinear effects of the present FV-FD model are similar to those in FNWD model. The shoaling process of a nonlinear solitary wave shows increased effect of nonlinearity while propagating into a region with shallower water depth. This case provides the numerical investigation in understanding the fission phenomena by presenting the deformation of the crest of the primary solitary waves and the formation of the secondary waves.

Another case studied by Madsen and Mei [33] is also selected to test the model performance in simulating the shoaling of a solitary wave over a sloping bottom. A solitary wave with amplitude $a=0.914 \mathrm{~cm}$ is simulated within a 6-m long domain including a 1:20 sloping bottom. The schematic diagram showing the domain and sloping bottom is given in Figure 6. Four gauging stations reported in Madsen and Mei [33] for the experimental measurements are marked as 1,2, 3, and 4 in Figure 6. They are located respectively at $\mathrm{x}=150,276.2,365.1$ and 451.46 $\mathrm{cm}$. The water depth changes from $\mathrm{h}_{0}=7.62 \mathrm{~cm}$ at the upstream domain to $\mathrm{h}_{1}=3.81 \mathrm{~cm}$ at the downstream region. The crest of the wave is located at $\mathrm{x}=-80 \mathrm{~cm}$, initially. Madsen and Mei [33] presented theoretical solutions and experimental observations for the time variation of the free-surface profiles. The results obtained from the present model are plotted in Figures $7 \mathrm{a}-7 \mathrm{~d}$ to compare to the theoretical solutions and experimental data given by Madsen and Mei [33]. The numerical results of Yuan and $\mathrm{Wu}[32]$ using a finite-difference model are also included in Figure 7 for comparisons. In Figure $7 \mathrm{a}$, the results at $\mathrm{t}=0$ correspond to the wave condition when the wave crest reaches the
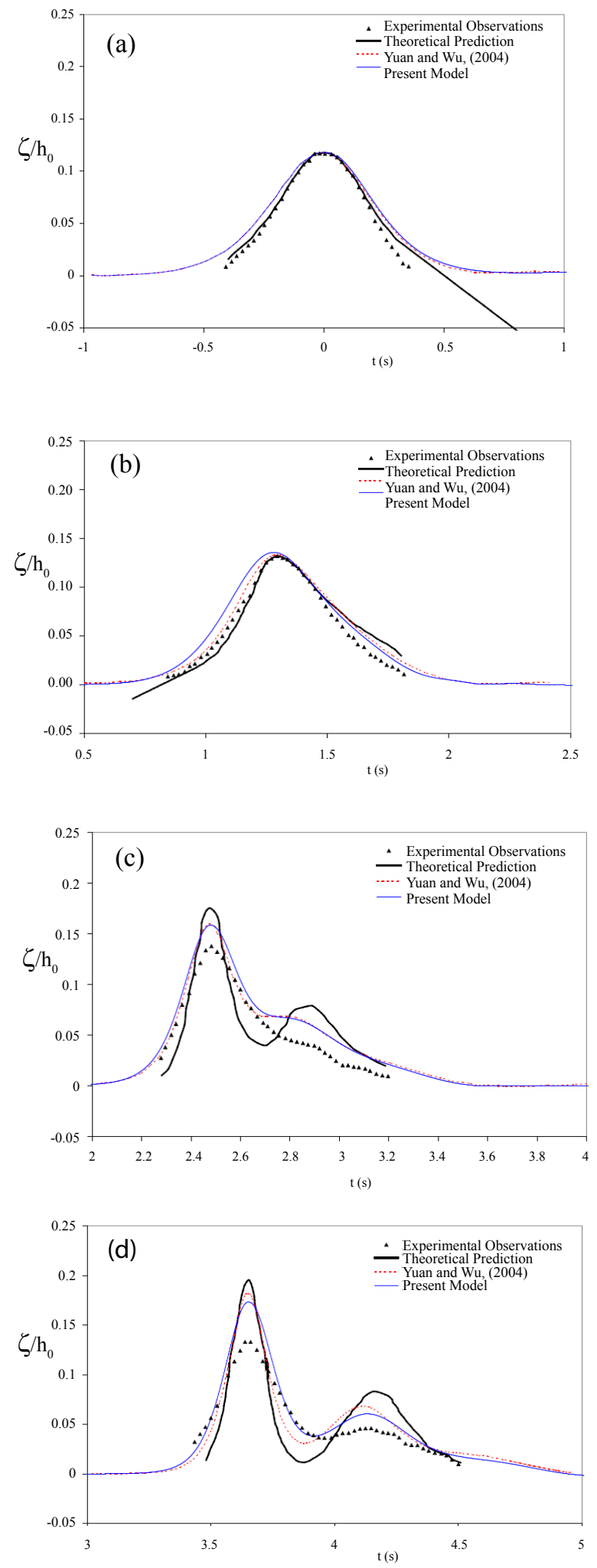

Figure 7: Comparisons of free-surface profiles among the theoretical, experimental results [33] numerical solutions by Yuan and $\mathrm{Wu}$ [32], and the present model solutions at gauging positions: (a) 1, (b) 2, (c) 3, (d) 4 . 
position 1. In Figure 7, it is noticed that the numerical results including those from the present model are shown to have a better agreement with measured data than the simplified but theoretically predicted values. The present numerical model performs reasonably well when compared to the results from the experimental measurements and numerical solutions by Yuan and $\mathrm{Wu}$ [32] especially at the positions 1 and 2. Both numerical models predict similar wave profiles and slightly overestimate the peak of the main wave at locations 3 (Figure $7(\mathrm{c})$ ). At location 4, the results are presented in Figure $7 \mathrm{~d}$. The present numerical model can provide slightly better predictions than those from Yuan and $\mathrm{Wu}[32]$ when comparing to the measured data. The formation of a secondary wave as a result of the shoaling effect is clearly shown in Figures $7 \mathrm{c}$ and $7 \mathrm{~d}$. The present solver is again demonstrated to be able to model reasonably well on the propagation and shoaling of a solitary wave over a region with a sloping bottom.

\section{Conclusions}

A one dimensional FV-FD free-surface flow solver is developed to simulate propagation of solitary waves in regions of different water depths and their head-on collisions. The extended Boussinesq equations by Madsen and Sorensen [3] are solved numerically by using the combined FV and FD methods, where FV is applied to discretize the conservative and source terms and FD is for the dispersive terms. Second order schemes and limiters are used to control unwanted small oscillations. The interaction of two solitary waves with equal-amplitude is simulated with the solver. The whole process of head-on collision including merging, interaction, and recovering back to their original shapes is presented with the plots showing the time variation of freesurface profiles. The maximum amplitude of the waves after completely merging is found to be close to the analytical results. Also, comparisons of the present model results with those of WNWD and FNWD models developed by Zhong and Wang [25] suggest the good performance received by the present model. A solitary wave propagating over a sloping bottom is also simulated. The fission process is verified with the present solver after the solitary wave interacts with the variable bottom. As a solitary wave propagates over a sloping bottom and reaches the shallower water depth region, the main wave separates into a primary wave followed with a sequence of secondary waves with decreasing wave amplitudes. The wave profiles computed with the present solver at different times agree well with those obtained by Zhong and Wang [25]. Overall, the present FV-FD solver is demonstrated to be capable of modeling interactions of solitary waves and simulating fission process over domains of variable depth.

\section{References}

1. Boussinesq PJ (1872) Theorie des ondes et des ramous qui se propagent le long d'un canal rectangulaire horizontal. Journal de Mathematique Pures et Appliquees, Deuxieme Serie 17: 55-108.

2. Madsen PA, Murray R, Sorensen OR (1991) A new form of the Boussinesq equations with improved linear dispersion characteristics. Coastal Eng 15: 371388.

3. Madsen PA, Sorensen OR (1992) A new form of the Boussinesq equations with improved linear dispersion characteristics, Part 2, A slowly-varying bathmetry. Coastal Eng 18: 183-204

4. Wu TY (1981) Long waves in ocean and coastal waters. J Engrg Mech Div ASCE 107: 501-522.

5. Nwogu O (1993) Alternative form of Boussinesq equations for near shore wave propagation. J of Waterway, Port, Coastal, and Ocean Eng 119: 618-638.

6. Peregrine DH (1967) Long waves on a beach. J Fluid Mech 27: 815-827.

7. Abbott M, McCowan AD, Warren IR (1984) Accuracy of short-wave numerical models. J Hydr Eng 110: 1287-1301.
8. Wang KH, Li W, Lee HS (2008) Propagation and Transformation of Periodic Nonlinear Shallow-Water Waves in Basins with Selected Breakwater Systems. Computers and Fluids 37: 931-942.

9. Zhong Z, Wang KH (2008) Time-accurate stabilized finite-element model for weakly nonlinear and weakly dispersive water waves. Int J Numer Meth Fluids 57: 715-744

10. Godunov S (1959) A divergence scheme for numerical computation of discontinuous solution of hydrodynamic equations. Math Sbornik 43: 271-306.

11. Anastasiou K, Chan CT (1997) Solution of the 2D shallow water equations using the finite volume method on unstructured triangular meshes. Int $\mathrm{J}$ Numer Meth Fluids 24: 1225-1245

12. Brufau P, Garcia-Navarro P, Vazquez-Cendon ME (2004) Zero mass error using unsteady wetting-drying conditions in shallow flows over dry irregular topography. Int J Numer Meth Fluids 45: 1047-1082.

13. LeVeque RJ (1998) Balancing source terms and flux gradients in highresolution methods: the quasi-steady wave-propagation algorithm. J Comp Phys 146: 346-365.

14. Stansby PK (2003) Solitary wave run up and overtopping by a semi-implicit finite volume shallow-water Boussinesq model. J Hydr Res 41: 639-647.

15. Erduran KS, Ilic S, Kutija V (2005) Hybrid finite-volume finite-difference scheme for the solution of Boussinesq equations. Int J Numer Meth Fluids 49: 12131232.

16. Shiach JB, Mingham CG (2009) A temporally second-order accurate Godunov type scheme for solving the extended Boussinesq equations. Coast Eng 56 $32-45$.

17. Tonelli M, Petti M (2010) Finite volume scheme for the solution of 2D extended Boussinesq equations in the surf zone. Ocean Engineering 37: 567-582.

18. Kazolea M, Delis AI, Nikolos IK, Synolakis CE (2012) An unstructured finite volume numerical scheme for extended 2D Boussinesq-type equations. Coastal Engineering 69: 42-66.

19. Bowler S (1991) The life of a solitary wave. New Scientist Magazine.

20. Byatt-Smith JGB (1971) An integral equation for unsteady surface waves and a comment on Boussinesq equation. J Fluid Mech 49: 625-633.

21. Yih CS, Wu TY (1995) General solution for interaction of solitary waves including head-on collisions. Acta Mechanica Sinica 11: 193-199.

22. Hammack J, Henderson D, Guyenne P, Yi M (2005) Solitary wave collisions. Advances in Engineering Mechanics and Outlooks in Honor of Theodore Y-T Wu World Scientific 173-194.

23. Grilli ST, Subramanya R, Svendsen IA, Veeramony J (1994) Shoaling of solitary waves on plane beaches. J Waterw Port, Coastal, and Ocean Eng 120: 609-628.

24. Zhou JG, Causon DM, Mingham CG, Ingram DM (2001) The surface gradient method for the treatment of source terms in the shallow-water equations. J Comp Phys 168: 1-25.

25. Zhong Z, Wang KH (2009) Modeling Fully Nonlinear Shallow-Water Waves and Their Interactions with Cylindrical Structures. Computers \& Fluids 38: 10181025.

26. Roe PL (1981) Approximate Riemann solvers, parameter vectors, and difference schemes. J Comp Phys 43: 357-372.

27. van Leer B (1979) Towards the ultimate conservative difference scheme $V A$ second order sequel to Godunov's method. J Comp Phys 32: 101-136

28. Bradford SF, Sanders BF (2005) Performance of high resolution, non-level bed, shallow water models. J Eng Mech 131: 1073-1081.

29. van Leer B (2006) Upwind and High-Resolution Methods for Compressible Flow: From Donor Cell to Residual-Distribution Schemes. Communications in Computational Physics 1: 192-206.

30. Roe PL (1986) Characteristic-based schemes for the Euler equations. Ann Rev Fluid Mech 18: 337-365.

31. Wei G, Kirby JT, Grilli S, Subramanya R (1995) A Fully Nonlinear Boussinesq Model for Surface Waves, Part 1 Highly Nonlinear Unsteady Waves. J Fluid Mech 294: 71-92. 
Citation: Wang KH, Turan B (2014) Modeling Interactions and Shoaling of Solitary Waves Using a Hybrid Finite Volume and Finite Difference Solver. J Appl Computat Math 3: 173 doi:10.4172/2168-9679.1000173

Page 8 of 8

32. Yuan $\mathrm{H}, \mathrm{Wu} \mathrm{CH}$ (2004) A two-dimensional vertical non-hydrostatic $\sigma$ model with an implicit method for free-surface flows. Int J Numer Meth Fluids 44: 811-835.
33. Madsen OS, Mei CC (1969) The transformation of a solitary wave over an uneven bottom. J Fluid Mech 39: 781-791. 